\title{
Graphene-induced spatial charge separation for selective water splitting over $\mathrm{TiO}_{2}$ photocatalyst
}

\author{
Shixiong Min ${ }^{\mathrm{a}, *}$, Fang Wang ${ }^{\mathrm{a}}$, Gongxuan $\mathrm{Lu}^{\mathrm{b}, *}$ \\ ${ }^{a}$ School of Chemistry and Chemical Engineering, Beifang University of Nationalities, Yinchuan, 750021, \\ Ningxia Province, China. \\ ${ }^{b}$ State Key Laboratory for Oxo Synthesis and Selective Oxidation, Lanzhou Institute of Chemical Physics, \\ Chinese Academy of Sciences, Lanzhou, 730000, China. \\ *Corresponding author.
}

Fax: 86-951-2067915; Tel: 86-951-2067917; E-mail: msxwf@163.com (S. Min).

Fax/Tel: 86-93-4968178. E-mail: gxlu@lzb.ac.cn, gxlu@ns.lzb.ac.cn (G. Lu). 


\begin{abstract}
Here we report that separately decorating $\mathrm{TiO}_{2}$ (P25) nanoparticles and $\mathrm{CoO}_{\mathrm{x}}$ cocatalyst on single reduced graphene oxide (rGO) sheet results in an advanced photocatalyst, P25-rGO-Co, which exhibits enhanced photocatalytic performances for selective $\mathrm{H}_{2}$ or $\mathrm{O}_{2}$ evolution from water splitting. Characterization results show that rGO cannot only efficiently accept and transport photogenerated electrons but also shuttle holes to $\mathrm{CoO}_{\mathrm{x}}$ cocatalyst simultaneously, achieving an efficient spatial charge separation on P25-rGO-Co, thus improving the half-reaction efficiency.
\end{abstract}

Keywords: reduced graphene oxide; $\mathrm{TiO}_{2}$; spatial charge seperation; water splitting 


\section{Introduction}

Graphene, as a promising two-dimensional (2D) carbon nanomaterial, has been incorporated with the semiconductors to enhance the photocatalytic and photoelectrochemical performances of semiconductorbased composite materials [1,2]. For examples, nanocomposites of graphene with semiconductors have shown enhanced activity for $\mathrm{H}_{2}$ or $\mathrm{O}_{2}$ evolution reaction [3,4]. It has also been shown that the graphene can efficiently capture and shuttle the electrons form excited dye to decorated $\mathrm{H}_{2}$-evolving cocatalysts [57], improving the $\mathrm{H}_{2}$ evolution activity in dye sensitized systems. Among above-mentioned studies, it has been generally accepted that the graphene mainly offers a rapid 2D electron transportation pathway to achieve an efficient separation of photogenerated charge carriers due to its excellent electron accepting and transporting properties. However, the role of graphene for transporting photogenerated holes has not been sufficiently addressed in the field of photocatalytic water splitting, although it has been demonstrated by Gemi et al. [1] that holes have almost identical mobility to electron on graphene sheets. In addition, above studies only showed the applications of anchoring single-nanoparticle components on graphene for $\mathrm{H}_{2}$ evolution, but decorating two even more (co)catalyst components on a single graphene sheet that can carry out selective catalytic reactions with enhanced performances at separated active sites has not been explored [8,9].

Herein, we report an advanced P25-rGO-Co photocatalyst prepared by separately decorating $\mathrm{TiO}_{2}$ (P25) and $\mathrm{CoO}_{\mathrm{x}}$ species on a single reduced graphene oxide (RGO) sheet via a two-step hydrolysishydrothermal method. Experimental results reveal that the presence of rGO not only provides an ideal 2D matrix for spatially anchoring $\mathrm{P} 25$ and $\mathrm{CoO}_{\mathrm{x}}$ cocatalyst with high dispersion, but also offers a rapid transportation pathway for both photogenerated electrons and holes, thus a efficient spatial charge seperation and an enhanced photocatalytic $\mathrm{H}_{2}$ and $\mathrm{O}_{2}$ evolution activity are acheved on P25-rGO-Co photocatalyst.

\section{Experimental}

\subsection{Photocatalyst preparation}


The P25-rGO-Co photocatalyst was synthesized by separately decorating $\mathrm{CoO}_{\mathrm{x}}$ species and P25 nanoparticles on rGO sheets using two-step hydrolysis-hydrothermal method (Fig.S1). Typically, 20 mg of GO was dispersed in $35 \mathrm{~mL}$ of ethanol, to which $2.0 \mathrm{~mL}$ of $10 \mathrm{mg} / \mathrm{mL} \operatorname{Co}(\mathrm{Ac})_{2}$ aqueous solution was added to form GO-Co ${ }^{2+}$ complex. Next, $0.5 \mathrm{~mL}$ of $30 \% \mathrm{NH}_{4} \mathrm{OH}$ was added to hydrolysis $\mathrm{Co}^{2+}$ to form $\mathrm{CoO}_{\mathrm{x}}$ species on GO sheets at $353 \mathrm{~K}$. After stirring for $10 \mathrm{~h}, 1.0 \mathrm{~g}$ of $\mathrm{TiO}_{2}$ (P25) was added into above mixture and sonicated for $15 \mathrm{~min}$ and the reaction mixture was hydrothermally heated at $423 \mathrm{~K}$ for $3 \mathrm{~h}$. The products were collected by centrifugation and washed with ethanol and water, and dried in air. The obtained sample was denoted as P25-rGO-Co. P25-Co and P25-rGO photocatalysts were prepared under the same experimental conditions without adding GO and Co salt, respectively.

\subsection{Characterizations}

Transmission electron microscopy (TEM) and high-resolution TEM (HRTEM) images were taken with a Tecnai-G ${ }^{2}$-F30 field emission transmission electron microscope. X-ray diffraction (XRD) patterns of the samples were recorded on a Rigaku B/Max-RB diffractometer with a nickel filtrated $\mathrm{Cu} K \alpha$ radiation operated at $40 \mathrm{kV}$ and $40 \mathrm{~mA}$. X-ray photoelectron spectroscopy (XPS) measurements were performed on

a K-Alpha-surface Analysis (Thermon Scientific) using X-ray monochromatization. Steady-state and time-resolved photoluminescence spectra were recorded with a Horiba Scientific FluoroMax-4 spectrofluorometer.

\subsection{Photocatalytic experiments}

Photocatalytic experiments were performed in a sealed quartz flask $(150 \mathrm{~mL})$ with a flat window and a silicone rubber septum for sampling. The light source was a 250-W high pressure mercury lamp. For $\mathrm{H}_{2}$ evolution experiments, $50 \mathrm{mg}$ of the photocatalyst was dispersed in $80 \mathrm{~mL}$ of $20 \%(\mathrm{v} / \mathrm{v}) \mathrm{CH}_{3} \mathrm{OH}$ aqueous solution. For $\mathrm{O}_{2}$ evolution experiments, $50 \mathrm{mg}$ of the photocatalyst was dispersed in $80 \mathrm{~mL}$ of $0.05 \mathrm{M}$ $\mathrm{AgNO}_{3}$ solution containing $0.2 \mathrm{~g} \mathrm{La}_{2} \mathrm{O}_{3}$. The reaction mixture was sonicated for $5 \mathrm{~min}$ and degassed by bubbling Ar gas for 40 min prior to light irradiation. The amount of $\mathrm{H}_{2}$ and $\mathrm{O}_{2}$ evolution was analysed using Agilent 6820 gas chromatography (TCD, 13X column, Ar). 


\subsection{Photoelectrochemical measurements}

Photoelectrochemical measurements were carried out with an electrochemical analyser (CHI660A) in a standard three-compartment cell. Pt foil was used as counter electrode and a saturated calomel electrode (SCE) as the reference electrode. The supporting electrolyte was $0.5 \mathrm{M} \mathrm{Na}_{2} \mathrm{SO}_{4}$ solution. A $250 \mathrm{~W}$ high pressure mercury lamp was used as light source. The working electrodes were prepared by drop-coating $0.2 \mathrm{~mL}$ of sample suspensions $(3.57 \mathrm{mg} / \mathrm{mL})$ directly onto the conductive surface $\left(1.6 \mathrm{~cm}^{2}\right)$ of ITO glass.

\section{Results and discussions}

Fig. 1a shows the TEM image of the P25-rGO-Co photocatalyst, in which the P25 nanoparticles (NPs) are decorated throughout rGO sheets and no Co species are observable on its surface. High-resolution TEM in Fig. 1b reveals that no crystalline Co species could be observed on rGO surface, but HAADF image and EDX mappings in Fig. 1c reveal that the Co species distribute thoughout rGO sheets. This result suggests that the loaded Co species is most likely amorphous and highly dispersed on rGO sheets, which is further comfirmed by the EDX profiles in Fig. 1d. EDX profile recorded at the middle of the rGO sheet (spot 1) only shows C, O, and Co signals, while EDX profile collected at aggregated P25 NPs (spot 2) presents strong C, O, and Ti signals but without Co signals, suggesting the spatial anchoring of Co species and P25 NPs on single rGO sheets. The P25-rGO-Co, P25-Co, and P25-rGO exhibit similar XRD patterns (Fig. S2) to P25 and there are no extra diffraction peaks for the rGO and Co species. XPS confirms the reduction of GO to rGO with $\sim 4$ at. \% N doping (Fig. S3a and b). Co2p XPS spectra (Fig. S3c) show two major peaks at 796.7 and $780.9 \mathrm{eV}$, corresponding to Co2 $\mathrm{p}_{1 / 2}$ and Co2 $\mathrm{p}_{3 / 2}$ of the $\mathrm{Co}^{3+}$ and

$\mathrm{Co}^{2+}$ oxidation states, respectively, with a weak satellite peak at $790.7 \mathrm{eV}$ related to the $\mathrm{Co}^{2+}$ components [10]. These results reveal that the loaded Co species contains both $\mathrm{Co}^{2+}$ and $\mathrm{Co}^{3+}$ oxides (referred to as $\mathrm{CoO}_{\mathrm{x}}$ hereafter). Therefore, above results strongly confirm that the rGO serves as an ideal matrix to spatially anchor P25 NPs and $\mathrm{CoO}_{x}$ species on single 2D rGO sheet.

The photocatalytic $\mathrm{H}_{2}$ evolution activities of various photocatalysts were displayed in Fig. 2a and b. P25 NPs alone has a low rate of $\mathrm{H}_{2}$ evolution, while the incorporation of rGO leads to a $\sim 2.5$-fold 
enhancement in $\mathrm{H}_{2}$ evolution rate for P25-rGO. Without rGO but loading $\mathrm{CoO}_{\mathrm{x}}$, P25-Co shows a much higher $\mathrm{H}_{2}$ evolution rate because $\mathrm{CoO}_{x}$ can facilitate the charge separation by efficiently capturing photogenerated holes [10]. Notably, after loading $\mathrm{CoO}_{x}$ on rGO, the rate of $\mathrm{H}_{2}$ evolution on P25-rGO-Co is further enhanced, which is even 1.6 times higher than P25-Co. On the other hand, the photocatalytic $\mathrm{O}_{2}$ evolution activities for above four photocatalysts were compared in Fig. 2c and d, where the P25-rGO-Co also shows the highest rate of $\mathrm{O}_{2}$ evolution. Note that the rGO itself is inactive for both $\mathrm{H}_{2}$ and $\mathrm{O}_{2}$ evolution reactions. For comparison, a photocatalyst of Co-P25-rGO was prepared, in which 1.5 nm $\mathrm{CoO}_{\mathrm{x}}$ was first loaded on P25 NPs (Co-P25) and then the Co-P25 was decorated on rGO sheets (Fig. S4). The Co-P25-rGO shows $\mathrm{H}_{2}$ and $\mathrm{O}_{2}$ evolution rates inferior to that of P25-rGO-Co (Fig. S5). These results indicate that the spatial anchoring of $\mathrm{CoO}_{\mathrm{x}}$ and P25 NPs on rGO sheets seems to be crucial for achieving enhanced photocatalytic performances on P25-rGO-Co.

In order clarify the essential roles of rGO and $\mathrm{CoO}_{\mathrm{x}}$ in enhancing the photocatalytic activity of P25rGO-Co, the transient photocurrent responses for photocatalysts were measured under UV light irradiation at zero bias, as shown Fig. 3a. It is clear to see that the P25 shows a very low photocurrent. With $\mathrm{CoO}_{\mathrm{x}}$ loading, the photocurrent of P25-Co doubles as compared to P25 because the loading of $\mathrm{CoO}_{\mathrm{x}}$ reduces the amount of holes accumulated and thus prohibits the surface charge recombination [10]. Furthermore, when P25 is decorated on rGO sheets, the photocurrent is further enhanced. Note that loading of $\mathrm{CoO}_{\mathrm{x}}$ leads to much less pronounced enhancement in photocurrent of P25 as compared to rGO, suggesting that although the capturing of photogenerated holes of $\mathrm{P} 25$ by $\mathrm{CoO}_{\mathrm{x}}$ species is feasible, the lower electron mobility of $\mathrm{CoO}_{\mathrm{x}}\left(5.0 \sim 7.0 \times 10^{-5} \mathrm{~cm}^{2} / \mathrm{Vs}\right)$ than $\mathrm{rGO}\left(2.0 \times 10^{6} \mathrm{~cm}^{2} / \mathrm{Vs}\right)$ increases the recombination between the electrons in P25 and the holes in $\mathrm{CoO}_{\mathrm{x}}$, resulting in much lower photocurrent for P25-Co. Notably, P25-rGO-Co exhibits a remarkably enhanced photocurrent, which is 20, 10, and 4 times higher than that of P25, P25-Co, and P25-rGO, respectively. Given that the decorated P25 NPs on rGO sheets were spatially separated from $\mathrm{CoO}_{x}$, the clearly enhanced photocurrent of P25-rGO-Co indicates that the rGO not only provides a fast transfer pathway for electrons [8], but also can shuttle 
holes to loaded $\mathrm{CoO}_{x}$ efficiently, resulting in a spatial charge separation in P25-rGO-Co. To further support this point, the steady-state (Fig. 3b) and time-resolved photoluminescence (Fig. 3c) spectra of photocatalysts were performed. The results show that the PL intensity for both P25-rGO-Co and P25-rGO is much weaker than that of P25-Co and P25, indicating that the direct charge recombination can be greatly suppressed. Meanwhile, the emission decay of the P25-rGO-Co and P25-rGO is slower than that of P25-Co and P25, suggesting that the enhanced charge separation is related to the prolonged lifetime of accumulated electrons and holes in P25-rGO-Co and P25-rGO [11].

In addition, the electrochemical activities of these photocatalysts were investigated, as shown in Fig. 3d. During the cathodic polarization process, the presence of $\mathrm{rGO}, \mathrm{CoO}_{\mathrm{x}}$, or both $\mathrm{rGO}$ and $\mathrm{CoO}_{\mathrm{x}}$ does not make a difference on the cathodic current density in the potential range of 0 to $-1.0 \mathrm{~V} v \mathrm{vs}$. SCE. This result implies that, in the case of P25-rGO-Co, the rGO is just an electron acceptor while the $\mathrm{CoO}_{\mathrm{x}}$ cocatalyst only act as a spectator for the hole transfer and a oxidation cocatalyst for electron donor, i.e., they are not involved in the reduction processes. On the other hand, during the anodic polarization process, the anodic current densities of P25-rGO-Co and P25-Co are nearly the same, but are obviously greater than those of P25-G and P25, unambiguously demonstrates that the loading of $\mathrm{CoO}_{\mathrm{x}}$ cocatalyst can greatly promote the hole transfer processes of P25 via rGO. In addition, the presence of rGO has a negligible effect on the anodic current density of P25 or P25-Co, indicating that the rGO involves in the oxidation processes just as a media for the hole transfer from the $\mathrm{P} 25$ to $\mathrm{CoO}_{\mathrm{x}}$ rather than an active sites for $\mathrm{O}_{2}$ evolution.

Based on the above analysis, a mechanism was proposed, as shown in Fig. 4. The conduction band minimum and valence band maximum of P25 are -0.29 and $2.9 \mathrm{~V}$ vs NHE, respectively, while the location of Femi level of rGO can be roughly estimated to $\sim 0 \mathrm{~V}$ vs NHE [11]. As a result, when the P25 and $\mathrm{CoO}_{\mathrm{x}}$ are separately decorated on rGO, a large energy offset between VB of P25 and rGO will form at the interface, while the energy offset between CB of P25 and rGO is relatively smaller. Once the electrons and holes are photogenerated in the P25, they will most likely separately transfer to the rGO 
owing to the formed energy band offsets [11], leading to a spatial charge separation on P25-rGO-Co, thus resulting in enhanced efficiencies for $\mathrm{H}_{2}$ and $\mathrm{O}_{2}$ evolution reactions.

\section{Conclusions}

An efficient photocatalyst, P25-rGO-Co, has been developed by spatially decorating P25 NPs and $\mathrm{CoO}_{\mathrm{x}}$ cocatalyst on single rGO sheet. Due to its high electron and hole mobility, the presence of rGO hardly involved in catalytic reactions but can significantly enhance the charge separation efficiency of $\mathrm{TiO}_{2}$ by capturing electrons and simultaneously transferring holes to $\mathrm{CoO}_{\mathrm{x}}$ on rGO sheets; as a result, the photocurrent response and photocatalytic activity of P25-rGO-Co towards $\mathrm{H}_{2}$ or $\mathrm{O}_{2}$ evolution was greatly enhanced as compared to reference samples.

\section{Acknowledgments}

This work is supported by the National Science Foundation of China (21463001, 21173242, 21263001), the Natural Science Foundation of the Ningxia Hui Autonomous Region (NZ15102), the Key project (2014XZZ02) and General project (2014XYZ02) of Beifang University of Nationalities, and the cultivation project of National Natural Science Foundation (2014QZP04).

\section{References}

[1] K. S. Novoselov, A. K. Geim, S. V. Morozov, D. Jiang, Y. Zhang, S. V. Dubonos, I. V. Grigorieva, A. A. Firsov, Science 306 (2004) 666-669.

[2] C. N. R. Rao, A. K. Sood, K. S. Subrahmanyam, A. Govindaraj, Angew. Chem. Int. Ed. 48 (2009) $7752-7777$.

[3] Q. J. Xiang, J. G. Yu, M. Jaroniec, Chem. Soc. Rev. 41 (2012) 782-796.

[4] X. Huang, X. Y. Qi, F. Boeyab, H. Zhang, Chem. Soc. Rev. 41 (2012) 666-686.

[5] S. X. Min, G. X. Lu, J. Phys. Chem. C 115 (2011) 13938-13945.

[6] S. X. Min, G. X. Lu, J. Phys. Chem. C 116 (2012) 25415-25424.

[7] S. X. Min, G. X. Lu, Int. J. Hydrogen Energy. 37 (2012) 10564-10574. 
[8] I. V. Lightcap, T. H. Kosel, P. V. Kamat, Nano Lett. 10 (2010) 577-583.

[9] P. V. Kamat, J. Phys. Chem. Lett. 1 (2010) 520-527.

[10] F. X. Zhang, A. Yamakata, K. Maeda, Y. Moriya, T. Takata, J. Kubota, K. Teshima, S. Oishi, K. Domen, J. Am. Chem. Soc. 134 (2012) 8348-8351.

[11] Y. Lin, K. Zhang, W. F. Chen, Y. D. Liu, Z. G. Geng, J. Zeng, N. Pan, L. F. Yan, X. P. Wang, J. G. Hou, ACS Nano 4 (2010) 3033-3038. 


\section{Figure Captions}

Fig. 1. (a) TEM image of P25-rGO-Co. (b) HRTEM image of selected area (blue rectangle) in panel a. (c) HAADF image and corresponding EDX element mappings. (d) EDX profiles recorded at selected spot 1 and 2 in panel c.

Fig. 2. (a, b) $\mathrm{H}_{2}$ and (c,d) $\mathrm{O}_{2}$ evolution activities over P25, P25-rGO, P25-Co, and P25-rGO-Co.

Fig. 3. (a) I-t profiles of photocatalyst electrodes in $0.5 \mathrm{M} \mathrm{Na}_{2} \mathrm{SO}_{4}$ solution under chopped UV light irradiation. (b) Steady-state (excitation wavelength: $280 \mathrm{~nm}$ ) and (c) time-resolved photoluminescence spectra (excitation wavelength: $280 \mathrm{~nm}$; detection wavelength: $410 \mathrm{~nm}$ ) of P25, P25-rGO, P25-Co, and P25-rGO-Co. (d) Linear sweep voltammetries (LSVs) of photocatalyst electrodes in $0.5 \mathrm{M} \mathrm{Na}_{2} \mathrm{SO}_{4}$ aqueous solution. The scan rate was $1 \mathrm{mV} / \mathrm{s}$.

Fig. 4. Proposed mechanism for selective $\mathrm{H}_{2}$ or $\mathrm{O}_{2}$ evolution from water splitting over P25-rGO-Co photocatalyst. 

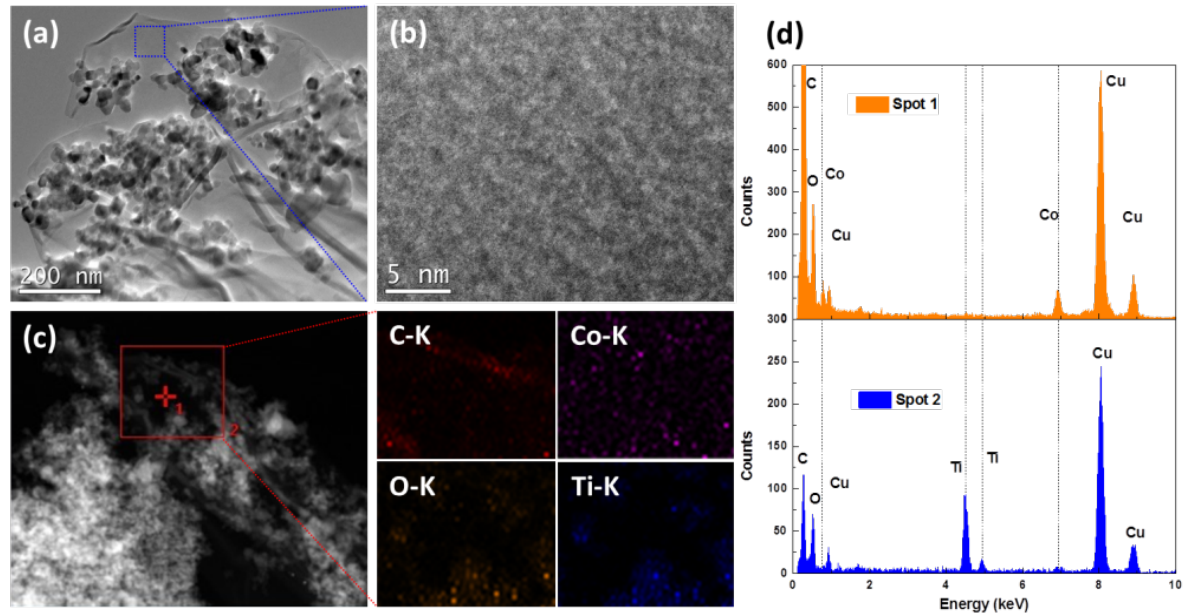

Fig. 1 

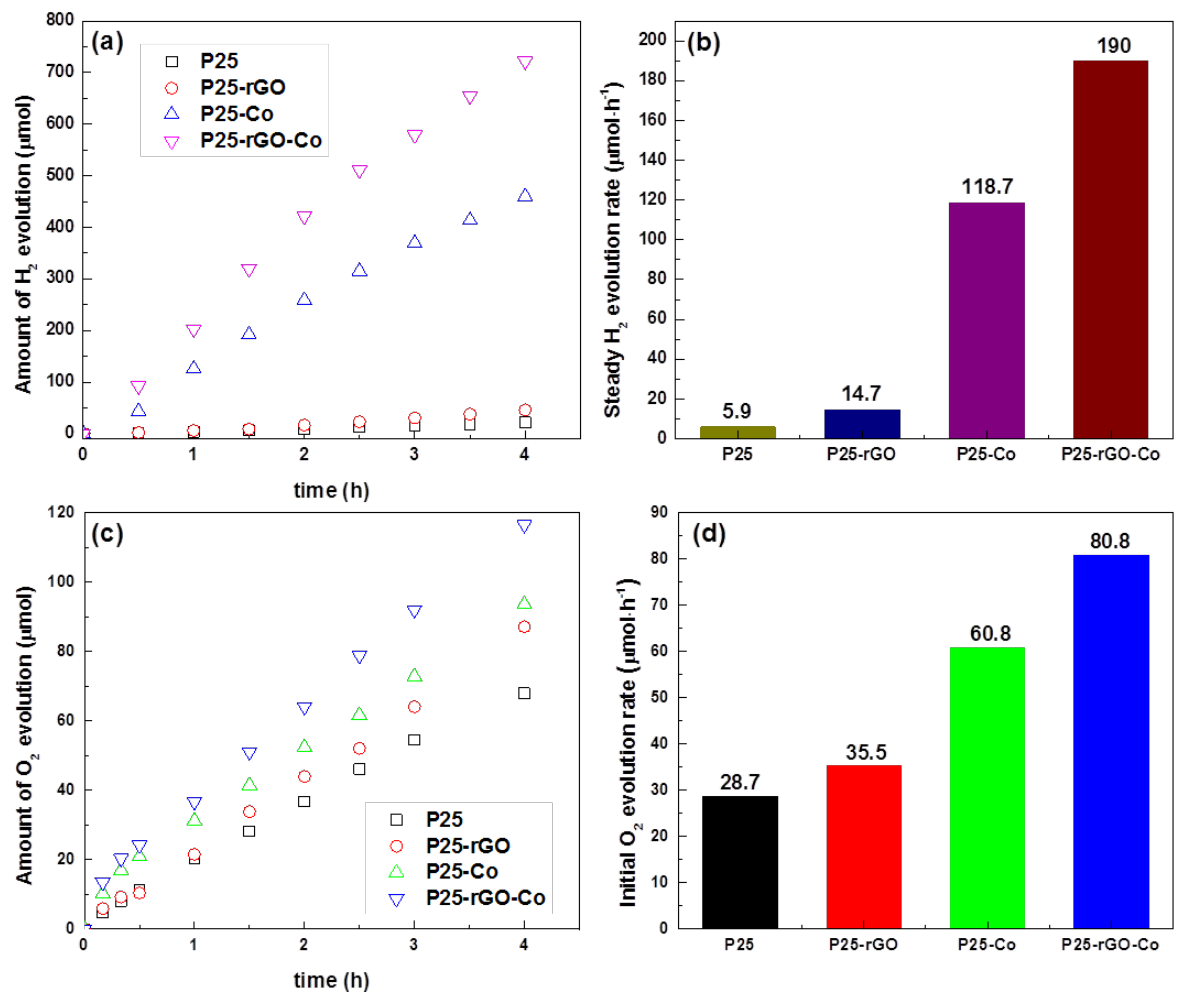

Fig. 2 

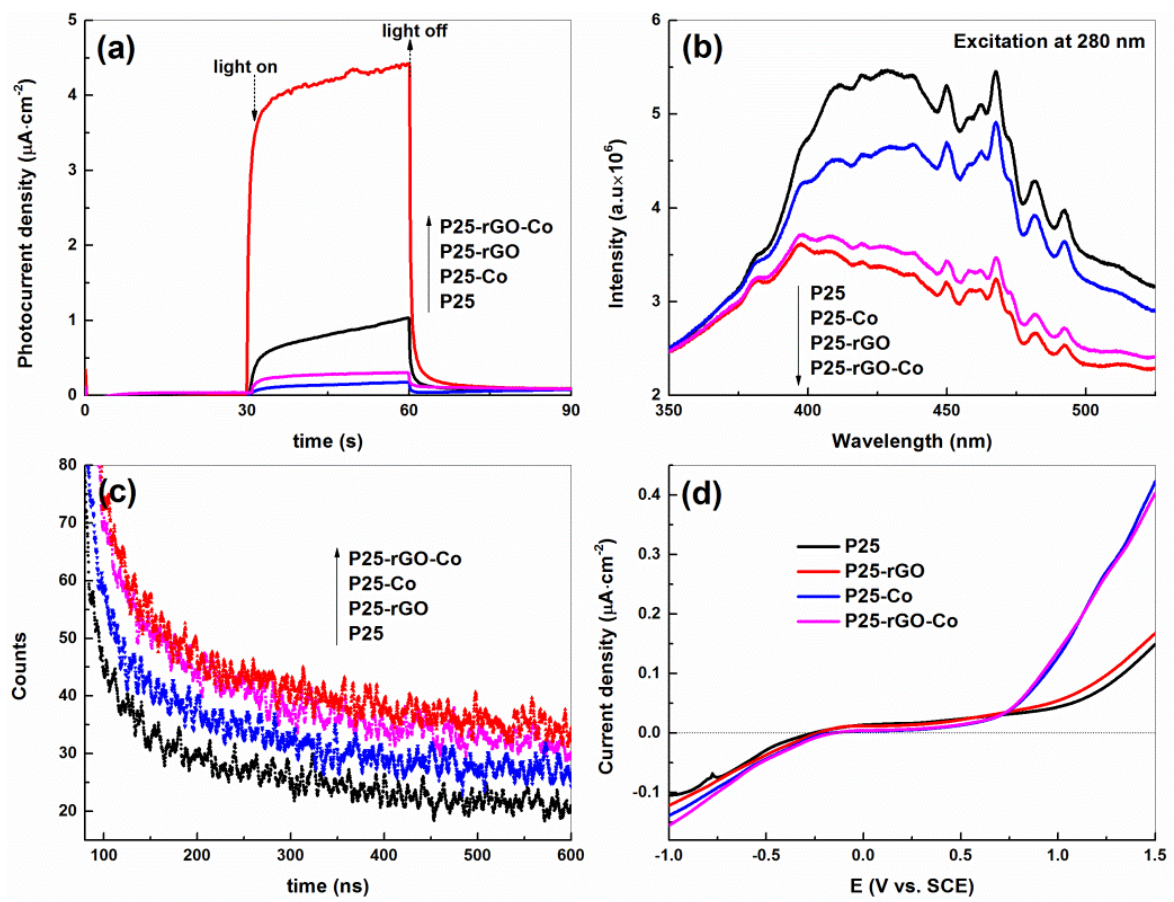

Fig. 3 


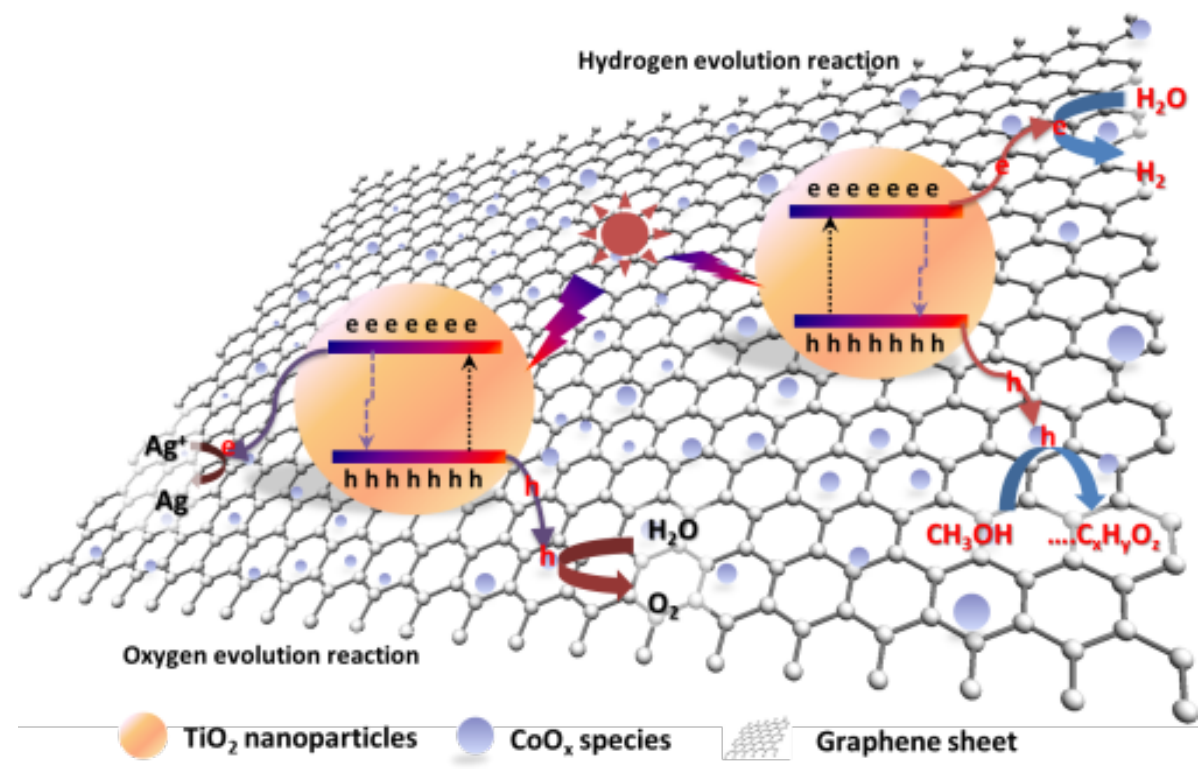

Fig. 4 


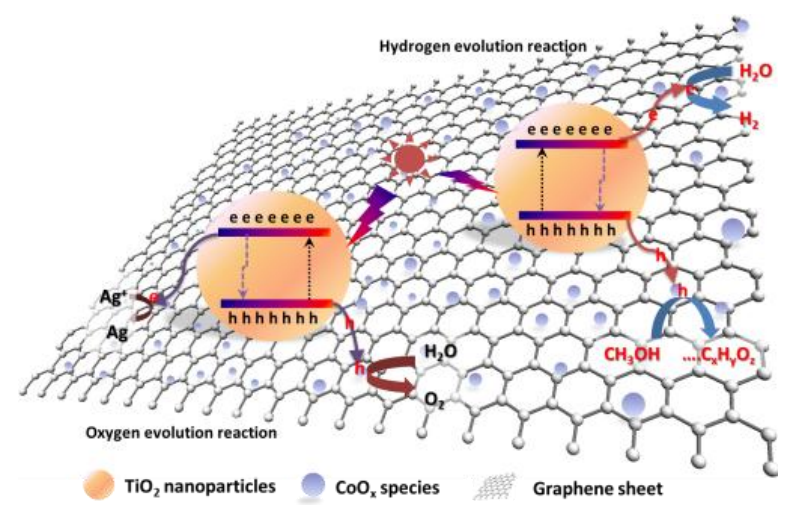

Graphical Abstract 